\title{
A Novel Tissue Protective Peptide: Cyclic Helix B Peptide
}

\author{
Bo Peng ${ }^{2,3}$ and Cheng Yang ${ }^{1,3}$ \\ ${ }^{1}$ Department of Plastic Surgery, Zhongshan Hospital, Fudan University, Shanghai, PR China \\ ${ }^{2}$ Department of Urology, Zhongshan Hospital, Fudan University, Shanghai, PR China \\ ${ }^{3}$ Shanghai Key Laboratory of Organ Transplantation, Shanghai, PR China
}

Corresponding author: Dr. Cheng Yang, Department of Plastic Surgery, Zhongshan Hospital, Fudan University; Shanghai Key Laboratory of Organ Transplantation, 180 Fenglin Road, Shanghai, 200032, PR China, Tel: +86-21-64041990; E-mail: esuperyc@163.com

Received: April 29, 2016; Accepted: May 13, 2016; Published: May 17, 2016

Citation: Peng B, Yang C. A Novel Tissue Protective Peptide: Cyclic Helix B Peptide. Transl Biomed. 2016, 7:2.

\section{Introduction}

Renal transplantation is one of the best choices for the treatment to the end stage renal disease. However, the shortage of the donor organs is a worldwide challenge, and the huge demand for transplantation forced the widely use of organs from expanded criteria donors or donation after cardiac death. Kidneys from these donors are highly sensitive to ischemia reperfusion (IR) injury, which might cause delayed graft function and affects both short- and long-term graft survival. Since IR injury is inevitable during kidney transplantation, it is urgent to develop an effective drug to optimize the organ preservation and ameliorate the tissue injury.

Erythropoietin (EPO) is an endogenous protein that exerts tissue-protective effects for a wide range of organs, but the very high dosage required to achieve that goal would cause unfavorable side effects, including hypertension and thrombosis [1-3]. Helix B surface peptide (HBSP), which was derived from the aqueous surface of the helix $B$ domain of EPO, was found to exert powerful tissue-protective function against IR injury without the ability of erythropoiesis $[4,5]$. However, this 11-amino acid protein is easily metabolized by the protease and its half-life is only 2-minute in vivo, which restricts its application [4].

Based on the structure of HBSP, our group firstly synthesized a novel proteolysis-resistant cyclic helix B peptide (CHBP) with improved metabolic stability and tissue-protective potency [6]. It was shown that CHBP significantly ameliorated inflammation in renal IR injury in a murine model, in terms of decreased apoptosis, pro-inflammatory cytokines expression and complement activation [6]. The main mechanism is the binding to the tissue protective receptor (TPR), which is a heterodimer receptor composed of EPO receptor (EPOR) and $\beta$ common receptor $(\beta c R)$, and then the downstream signaling pathway of Jak-2/STAT3 is activated [7,8]. Besides, we identified CHBPinduced autophagy through activation of AMPK and inhibition of mTOR complex 1 (mTORC1) in the IR injury kidney for the first time, which also contributed to its renoprotective effects [6]. We further proved that the administration of CHBP into preservation solution and autologous blood perfusate ameliorated IR injury in isolated porcine kidney by increasing renal blood flow and oxygenation and reducing apoptosis and inflammation, which suggested the promising application of CHBP in organ preservation [9].

IR injury of kidney is a well-established cause of renal fibrosis in long-term [10]. CHBP not only ameliorates the tissue injury of IR, but also attenuates fibrosis after IR through inhibition of TGF- $\beta$-induced extracellular matrix protein expression and epithelial-mesenchymal translation in tubular epithelial cells. The mechanism mainly involves the suppression of PI3K/Akt pathway and subsequent inhibition of FoxO3a [11]. In addition, CHBP also plays a role in immune regulation. It was revealed that $\mathrm{CHBP}$ suppressed dendritic cell (DC) maturation via the activation of Jak-2/STAT3/SOCS1 signaling pathway, which subsequently inhibited the activation of toll-like receptor (TLR). As a result, the CHPB-induced immature $D C$ ameliorated acute rejection in a rat kidney transplantation model [8].

Actually, IR injury is not confined to the transplantation, but quite common in many pathophysiologic situations, including acute kidney injury, acute coronary syndrome etc. Since the multiple tissue-protective functions of $\mathrm{CHBP}$, it is a promising drug in many fields. What's more, the evolution from EPO to CHBP results from the creative innovation, and shares the scientific methods with other drug development.

\section{Reference}

1. Hu L, Yang C, Zhao T, Xu M, Tang Q, et al. (2012) Erythropoietin ameliorates renal ischemia and reperfusion injury via inhibiting tubulointerstitial inflammation. J Surg Res 176: 260-266.

2. Brines M, Cerami A (2008) Erythropoietin-mediated tissue protection: reducing collateral damage from the primary injury response. J Intern Med 264: 405-432.

3. Lippi G, Franchini M, Favaloro EJ (2010) Thrombotic complications of erythropoiesis-stimulating agents. Semin Thromb Hemost 36: 537-549.

4. Brines $M$, Patel NS, Villa $P$, Brines $C$, Mennini $T$, et al. (2008) Nonerythropoietic, tissue-protective peptides derived from the tertiary structure of erythropoietin. Proc Natl Acad Sci USA 105: 10925-10930.

5. Yang C, Zhao T, Lin M, Zhao Z, Hu L, et al. (2013) Helix B surface peptide administered after insult of ischemia 
reperfusion improved renal function, structure and apoptosis through beta common receptor/erythropoietin receptor and PI3K/Akt pathway in a murine model. Exp Biol Med 238: 111-119.

6. Yang C, Xu Z, Zhao Z, Li L, Zhao T, et al. (2014) A novel proteolysis-resistant cyclic helix $\mathrm{B}$ peptide ameliorates kidney ischemia reperfusion injury. Biochim Biophys Acta 1842: 2306-2317.

7. Brines M, Cerami A (2012) The receptor that tames the innate immune response. Mol Med 18: 486-496.

8. Yang C, Zhang Y, Wang J, Li L, Wang L, et al. (2015) A novel cyclic helix $B$ peptide inhibits dendritic cell maturation during amelioration of acute kidney graft rejection through Jak-2/STAT3/SOCS1. Cell Death Dis 6: e1993.
9. Yang C, Hosgood SA, Meeta P, Long Y, Zhu T, et al. (2015) Cyclic Helix B Peptide in Preservation Solution and Autologous Blood Perfusate Ameliorates IschemiaReperfusion Injury in Isolated Porcine Kidneys. Transplantation direct 1: e6.

10. Bonventre JV, Yang L (2011) Cellular pathophysiology of ischemic acute kidney injury. J Clin Invest 121: 4210-4221.

11. Yang C, Cao Y, Zhang Y, Li L, Xu M, et al. (2015) Cyclic helix $B$ peptide inhibits ischemia reperfusion-induced renal fibrosis via the PI3K/Akt/FoxO3a pathway. J Transl Med 13: 355. 